\title{
Multiphoton dissociation for coherent and incoherent fields
}

\author{
S. Miret-Artés \\ Instituto de Matemáticas y Física Fundamental, Consejo Superior de Investigaciones Científicas, Serrano 123, 28006 Madrid, Spain \\ D. A. Micha \\ Quantum Theory Project, University of Florida, Gainesvillle, Florida 32611
}

(Received 29 June 1993)

\begin{abstract}
Photodissociation probabilities for coherent and incoherent laser sources are described, using numberand coherent-state representations of the light field. Previous calculations of $\mathrm{H}_{2}{ }^{+}$photodissociation in strong laser fields are used to calculate probabilities, for coherent and incoherent sources, that show increasing differences at lower light intensities.
\end{abstract}

PACS number(s): 42.50.Hz, 33.80.Wz, 33.80.Gj

The purpose of this work is to derive photodissociation probabilities in intense fields, corresponding to coherent and incoherent laser sources, in order to stress the importance of using the correct statistical averaging over photon source properties, when comparing theoretical results with experimental values.

Molecular photodissociation by intense laser fields is being actively studied. New effects have been predicted and observed, such as above-threshold dissociation (ATD), bond softening, and vibrational trapping [1-4], and an appropriate theoretical framework has been derived to understand these new phenomena. Usually, phenomena involving coherent photon absorption and stimulated emission have been described by a semiclassical time-dependent Hamiltonian with a uniform classical electric field $E_{0} \cos (\omega t+\phi)$ in the dipole-coupling approximation and have been combined with an expansion in molecular states, leading to a time-independent description of photodissociation in terms of Floquet blocks [5]. This approach has the advantage of providing simple and intuitive explanations of those phenomena and convenient close-coupling (CC) equations. Several methods for solving such CC equations can be found in the literature $[4,6,7]$. Also, wave-packet propagation methods $[3,8]$ have been applied to the study of molecular photodissociation, and a collisional time-correlation-function approach to the interaction of photons with polyatomic systems has been proposed for general laser sources [9].

Obviously, the number of CC equations to be solved increases dramatically with the intensity of the laser. The semiclassical description of the laser field is particularly convenient at high intensities [10]. Lasers operating above their thresholds are described by a superposition of occupation-number states; that is, they are not in a pure photon-number state. The greater the average occupation number, the closer is the approach to the one in terms of a classical electromagnetic field. For these cases, the statistical distribution of both the amplitude and the phase of the field can play a very important role in the final distribution of the fragments. To study their role, it is advantageous to begin with the coherent-state representation of the field [11]. Since the modes of the field are independent, only a single mode of the field will be considered in order to simplify the discussion. This approach was introduced some time ago $[12,13]$ within the rotating-wave approximation for weak intensities. Our approach avoids that approximation and, therefore, no general analytical solution is provided.

Our starting point is to use the theory developed in Ref. [4], as originally suggested [14]. This procedure is a generalization of the artificial-channel method [15] for the calculation of direct photodissociation cross sections in weak-field situations. In particular, two artificial channels are introduced in this formalism. The first one, a continuum channel, is added in order to transform the half-collision process into a full collisional one. The second one, a bound channel, plays the role of the true initial unperturbed molecular state, weakly coupled to the total molecular-field manifold. In other words, this bound channel is introduced in order to select the initial state of the molecular system. For radiative lifetimes shorter than pulse lengths, we can obtain the total photodissociation probability for a transition from an initial bound state $[|g\rangle$, with given vibrational and rotational numbers $(v, j)]$ to a continuum state $(|d\rangle$, with a given rotational number $j^{\prime}$ and a relative kinetic energy $\varepsilon$ ). Only two electronic states are considered. Thus, we have [4]

$$
P_{d n_{f} ; g v n_{i}}^{\mathrm{coh}}\left(E_{0}, \omega\right)=\int d \varepsilon \frac{\left|\left\langle d, \varepsilon, n_{f}|T| g, v, n_{i}\right\rangle\right|^{2}}{\left(\varepsilon-E_{g v}-n_{i} \hbar \omega\right)^{2}}
$$

where we have denoted to the left that the CC probabilities include coherence effects. Here $T$ is the transition operator of scattering theory for photon projectiles. The rotational quantum numbers have been dropped here, as any change in the rotation quantum number $j$ will be neglected. In Eq. (1), $n_{f}$ and $n_{i}$ are the final and initial number of photons in the field and $E_{g v}$ the initial energy level. Finally, $\omega$ is the frequency of the laser field. The quantity in the integral can be obtained directly by solving the $\mathrm{CC}$ equations and it is related to an $S$ matrix in a very simple way [4].

Our next step is to change to the coherent representa- 
tion for the initial state of the field. By introducing the transformation between number and coherent states

$$
\langle n \mid \alpha\rangle=\exp \left(-|\alpha|^{2} / 2\right) \alpha^{n} / \sqrt{n !},
$$

where $\alpha$ is the complex amplitude of a coherent electric field of magnitude $|\alpha|$ and phase $\phi$, the matrix elements of the $T$ operator can be expressed as

$$
\left\langle d, \varepsilon, n_{f}|T| g, v, \alpha_{i}\right\rangle=\sum_{n_{i}=0}^{\infty}\left\langle d, \varepsilon, n_{f}|T| g, v, n_{i}\right\rangle\left\langle n_{i} \mid \alpha_{i}\right\rangle .
$$

Averaging the absolute value square over the initial phase $\phi_{i}\left[\right.$ with $\left.\alpha_{i}=\left|\alpha_{i}\right| \exp \left(i \phi_{i}\right)\right]$, after a little algebra, we arrive at

$$
\begin{aligned}
\frac{1}{2 \pi} \int_{0}^{2 \pi} d \phi_{i} \mid & \left.\left\langle d, \varepsilon, n_{f}|T| g, v, \alpha_{i}\right\rangle\right|^{2} \\
& =\sum_{n_{i}=0}^{\infty}\left|\left\langle n_{i} \mid \alpha_{i}\right\rangle\right|^{2}\left|\left\langle d, \varepsilon, n_{f}|T| g, v, n_{i}\right\rangle\right|^{2},
\end{aligned}
$$

where, from Eq. (2), $\left|\left\langle n_{i} \mid \alpha_{i}\right\rangle\right|^{2}$ is a Poisson distribution.

Thus, from Eq. (4), the new transition probabilities for incoherent sources are obtained from CC calculations weighted by the Poisson distribution. This equation could be considered as a generalization of the work of Ref. [13]. Finally, from Eq. (1) averaged over the initial phase, the corresponding integral photodissociation probabilities are expressed as

$$
P_{d n_{f} ; g v\left|\alpha_{i}\right|}^{\mathrm{inc}}\left(E_{0}, \omega\right)=\sum_{n_{i}=0}^{\infty} P_{d n_{f} ; g v n_{i}}^{\mathrm{coh}}\left(E_{0}, \omega\right)\left|\left\langle n_{i} \mid \alpha_{i}\right\rangle\right|^{2} .
$$

As the final number of photons remaining in the field is usually not known, we have to sum over $n_{f}$ to obtain the final distribution of the fragments,

$$
P_{d ; g v\left|\alpha_{i}\right|}^{\text {inc }}\left(E_{0}, \omega\right)=\sum_{n_{f}=0}^{\infty} P_{d n_{f} ; g v\left|\alpha_{i}\right|}^{\text {inc }}\left(E_{0}, \omega\right) .
$$

This quantity $P_{d ; g v\left|\alpha_{i}\right|}^{\mathrm{inc}}\left(E_{0}, \omega\right)$ is the one to be compared to experimental results with incoherent light generated with very short pulses (for example, in the experiences in intense laser fields used in Ref. [2]). It depends on the value of $\left|\alpha_{i}\right|$, which can be extracted from a fitting procedure insofar as the intensity of the field can be expressed as $[10(b)]$

$$
I=\frac{c \hbar \omega}{V}\left|\alpha_{i}\right|^{2},
$$

where $V$ is the volume of the cavity.

For a wavelength of $329.7 \mathrm{~nm}$, the photodissociation of $\mathrm{H}_{2}^{+}$has been extensively studied for different intensities. Originally, the ATD process was predicted for that wavelength for which several theoretical studies could be found in the literature. Direct experimental observation has been reported in Ref. [2]. For this wavelength, we are very far from the maximum of absorption, and in a very off-resonant condition; consequently, the rotatingwave approximation cannot be applied. With the present formalism we can nevertheless very easily calculate pho- todissociation probabilities for any intensity of the field or alternatively for any $\left|\alpha_{i}\right|^{2}$. Assuming that for the experimental intensities $\Delta n=n_{i}-n_{f}=1,2,3$, we have that

$$
\begin{aligned}
P_{d ; g v\left|\alpha_{i}\right|}^{\mathrm{inc}}\left(E_{0}, \omega\right)= & P_{d ; g v}^{\mathrm{coh}, 1}\left(E_{0}, \omega\right) \sum_{n_{i}}\left|\left\langle n_{i} \mid \alpha_{i}\right\rangle\right|^{2} \\
& +P_{d ; g v}^{\mathrm{coh}, 2}\left(E_{0}, \omega\right) \sum_{n_{i}}\left|\left\langle n_{i} \mid \alpha_{i}\right\rangle\right|^{2} \\
& +P_{d ; g v}^{\mathrm{coh}, 3}\left(E_{0}, \omega\right) \sum_{n_{i}}\left|\left\langle n_{i} \mid \alpha_{i}\right\rangle\right|^{2},
\end{aligned}
$$

which can be reexpressed in terms of $\left\langle 0 \mid \alpha_{i}\right\rangle,\left\langle 1 \mid \alpha_{i}\right\rangle$, and $\left\langle 2 \mid \alpha_{i}\right\rangle$, using the completeness relation $\sum_{n_{i} \geq 0}\left|\left\langle n_{i} \mid \alpha_{i}\right\rangle\right|^{2}=1$. Thus, for $I=2.45 \times 10^{13} \mathrm{~W} / \mathrm{cm}^{2}$, experimental observation and $\mathrm{CC}$ calculations show that the photodissociation dynamics proceeds mainly via the absorption of two photons [2-4] and we can write, for $n_{i} \geq 2$,

$$
P_{d n_{f} ; g v n_{i}}^{\text {coh }}\left(E_{0}, \omega\right)=P_{d ; g v}^{\text {coh, } 2}\left(E_{0}, \omega\right) \delta_{n_{i}-n_{f}, 2} .
$$

Therefore, in the sum over $n_{i}$ in Eq. (5), only one term is retained, corresponding to $n_{i}-n_{f}=\Delta n=2$. Now, the sum over $n_{f}$ in Eq. (6) is reduced to

$$
\begin{aligned}
P_{d ; g v\left|\alpha_{i}\right|}^{\text {inc }}\left(E_{0}, \omega\right)=P_{d ; g v}^{\mathrm{coh}, 2}\left(E_{0}, \omega\right) & 1-\left|\left\langle 0 \mid \alpha_{i}\right\rangle\right|^{2} \\
& \left.-\left|\left\langle 1 \mid \alpha_{i}\right\rangle\right|^{2}\right) .
\end{aligned}
$$

We assume that $\left|\alpha_{i}\right|^{2} \simeq 9.0$ at that intensity. The corresponding volume of the cavity is then $633 \AA^{3}$, a reasonable value. The $\mathrm{CC}$ result is 0.993 , while the incoherence average is 0.989 . From this value of the volume and through Eq. (7), it is possible to calculate the $\left|\alpha_{i}\right|^{2}$ values for different intensities. Notice that here we have taken advantage of the particular relation $\Delta n=2$, valid at one wavelength and intensity. Generally $\Delta n \neq 2$.

In Table I, we show a comparison between the semiclassical results from $\mathrm{CC}$ calculations, which contain coherence effects, and those averaged by a Poisson distribution for incoherent light, for different intensities of the field and $\Delta n$ values. These $\mathrm{CC}$ results are obtained through the following relation between the matrix elements of the $T$ and $S$ operators of collision theory derived in Ref. [4] using the artificial channel procedure,

$$
\frac{\left|\left\langle d, \varepsilon, n_{f}|T| g, v, n_{i}\right\rangle\right|^{2}}{\left(\varepsilon-E_{g v}-n_{i} \hbar \omega\right)^{2}}=A\left|\left\langle d, \varepsilon, n_{f}|S| a\right\rangle\right|^{2},
$$

where $A$ is a constant determined by the calculations and $|a\rangle$ is the continuum artificial channel. In the same table, we have listed the $\left|\alpha_{i}\right|^{2}$ values and the probabilities for absorbing 1, 2, or 3 photons, respectively. As can be seen from this table, the differences in values are much more important as intensity decreases. This is clear evidence that the coherence of the laser field (included in the $\mathrm{CC}$ calculations) becomes important for intermediate and weak intensities with $\mathrm{cw}$ lasers. On the other hand, when the intensity increases, the average number of photons is high and the Poisson averaging modifies very little the semiclassical results, so that the coherence may be ig- 
TABLE I. Semiclassical probabilities from CC and phase-averaged results for four different intensities together with the corresponding value of $\left|\alpha_{i}\right|^{2}$. The columns correspond to fragmentation by absorption of one, two, or three photons, respectively.

\begin{tabular}{lcccc}
\hline$I\left(\mathrm{~W} / \mathrm{cm}^{2}\right)$ & $\left|\alpha_{i}\right|^{2}$ & CC Probabilities & Phase-averaged Probabilities & $\Delta n$ \\
\hline $5.6 \times 10^{13}$ & 20.68 & 0.320 & 0.319 & 1 \\
& & 0.667 & 0.666 & 2 \\
$2.45 \times 10^{13}$ & 9.0 & & & 1 \\
& & 0.993 & 0.989 & 2 \\
$7.0 \times 10^{12}$ & 2.60 & & & 1 \\
& & 0.795 & 0.583 & 2 \\
& \multirow{2}{*}{1.29} & 0.205 & 0.099 & 3 \\
$3.5 \times 10^{12}$ & & & & 1 \\
& & 0.554 & 0.205 & 2 \\
& & 0.446 & 0.063 & 3 \\
\hline \hline
\end{tabular}

nored. In other words, the physical origin of the probability decrease in Table $I$ is in the elimination of constructive phase interference when one averages over phases as shown in Eq. (4).

The discussion so far is appropriate for cw lasers. For laser pulses, the semiclassical approach must be changed accordingly. For such lasers, however, the one-mode description would still be valid, so that the main features of this paper are expected to apply to laser pulses.

\section{ACKNOWLEDGMENTS}

One of us (S.M.A.) would like to thank the Dirección General de Investigación Científica y Técnica of the Minister of Education and Science of Spain for support of a grant to collaborate at the University of Florida. The work of D.A.M. has been partly supported by the $\mathrm{Na}$ tional Science Foundation.
[1] A. Giusti-Suzor, X. He, O. Atabek, and F. H. Mies, Phys. Rev. Lett. 64, 515 (1990).

[2] P. H. Bucksbaum, A. Zavriyev, H. G. Muller, and D. W. Schumacher, Phys. Rev. Lett. 64, 1883 (1990); Phys. Rev. A 42, 5500 (1990); A. Zavriyev, P. H. Bucksbaum, J. Squier, and F. Saline, Phys. Rev. Lett. 70, 1077 (1993).

[3] A. Giusti-Suzor and F. H. Mies, Phys. Rev. Lett. 68, 3869 (1992).

[4] S. Miret-Artés, O. Atabek, and A. D. Bandrauk, Phys. Rev. A 45, 8056 (1992).

[5] S. I. Chu, J. Chem. Phys. 75, 2215 (1981).

[6] X. He, O. Atabek, and A. Giusti-Suzor, Phys. Rev. A 38, 5586 (1988); 42, 1585 (1990).

[7] D. A. Micha, J. Phys. Chem. 95, 8082 (1991).
[8] G. Jolicard and O. Atabek, Phys. Rev. A 46, 5845 (1992).

[9] D. Srivastava and D. A. Micha, Int. J. Quant. Chem: Quant. Chem. Symp. 21, 229 (1987).

[10] (a) W. H. Louisell, Quantum Statistical Properties of Radiation (Wiley, New York, 1973), Chap. V; (b) R. Loudon, The Quantum Theory of Light (Clarendon, Oxford, 1983), Chap. 8.

[11] R. J. Glauber, Phys. Rev. 98, 1692 (1955).

[12] B. R. Mollow, Phys. Rev. A 12, 1919 (1975).

[13] K. F. Freed and A. A. Villaeys, J. Chem. Phys. 70, 3071 (1979).

[14] O. Atabek and A. D. Bandrauk, Adv. Chem. Phys. 73, 825 (1989).

[15] M. Shapiro, J. Chem. Phys. 56, 2582 (1972). 